\title{
Alterations in lipid Composition during growth and senescence of Rauwolfia serpentina leaf
}

\author{
Poonam and Sanjay Mishra* \\ School of Biotechnology, IFTM University, Lodhipur Rajput, Delhi Road (NH-24), Moradabad 244102, U.P., \\ India \\ *Corresponding Author: Email: sanjaymishra@iftmuniversity.ac.in
}

\begin{abstract}
Various lipid classes and compounds were monitored during the period of leaf emergence to leaf drop of Rauwolfia serpentina. The expansion to early maturation phase was accompanied by cellular build-up of all major lipid classes, whilst aging and senescence were characterized by their significant turn down, except for the neutral lipids; the leaf monogalactosyl diglyceride/digalactosyl diglyceride ratio decreased from 4.6 (complete maturity) to 2.5 (abscised stage). The early maturation stage was the earliest stage when substantial amounts of free sterols and fatty acids could be pragmatic. The unsaturated/saturated fatty acid ratio was far lesser in the senescent leaf as compared to that of the fully expanded leaf. The specific changes in lipid composition may be evocative of simultaneous alterations in membrane ultrastructure and functions, putatively leading to perturbation of indole alkaloid impounding potential of the tissues of a pharmaceutically significant species.
\end{abstract}

Key words: Glycolipids, leaf development, phospholipids, Rauwolfia serpentina, senescence, sterol.

\section{Introduction}

Rauwolfia serpentina, (Apocynaceae) is a medicinal plant, commonly known as Indian snake root, chandra, sarpgandha, an evergreen, perennial, glabrous and erect under shrub. Generally it grows up to 15-45 $\mathrm{cm}$ in height, but may grow up to $90 \mathrm{~cm}$ under very favorable conditions. Leaves grow in whorls of 3-4, are deciduous, elliptic-lanceolate or obovate, pointed, green on the upper surface, pale-green underneath, 7.5 $\mathrm{cm}$ long and $3.5-5 \mathrm{~cm}$ broad in size, are aboriginal to India and Bangladesh and is found to grow wild in the Asian continent. It has been reported to contain 50 indole alkaloids that are mainly localized in the root bark (Klushnichenko et al., 1995). These alkaloids are, reserpine, yohimbine, serpentine, deserpidine, ajmalicine and ajmaline are used to treat hypertension (Von Poser . et al.,1990) and breast cancer (Stanford . et al., 1986), antidote against bite from poisonous reptiles, anti-dysentry (Bhatara et al.,1997). Reserpine, used as a natural tranquilizer was found to have several times greater hypotensive activity than the crude plant extract (Pullaiah et al.,2002). Rauwolfia root is reported to contain $0.7-3.0 \%$ of total alkaloids in the dry mass and the amount varies with time and source of collection (Kokate et al., 1998).

During the last decade, a lot of effort has been made with the view to exploring primary to secondary metabolite networks for indole alkaloid biosynthesis in C. roseus (Canel et al., 1998; Sangwan et al., 1998; van der Fits and Memelink, 2000; Ayora-Talavera et al., 2002; Hughes et al., 2002, 2004; Whitmer et al., 2002a,b). However, lipid metabolism together with qualitative and quantitative production and sequestration of medicinally and biotechnologically valuable phytochemicals like lipids in leaves of Rauwolfia serpentina is still a fast developing and promising area in which to work.

Lipids are basically structural and functional molecules of plant cells and tissues. They are known to affect certain membrane properties like permeability, fluidity and transport. In particular, lipids are sensitive to any change in physiological state as well as biotic and abiotic environments (Brown et al., 1987; Droillard et al., 1989; Duxbury et al., 1991; Paliyath and Droillard, 1992). The length and degree of unsaturation of fatty acids, the nature of sterols and the sterol/phospholipids ratio, collectively affect the fluidity of the membrane bilayer (Duxbury et al., 1991). Metabolically, certain alterations in lipid content and composition may be seen in relation to development of intracellular membranous compartmen-tation and the availability of intracellular (non-plasmic and periplasmic) spaces. Since vacuoles are intracellular spaces which function as accumulation sites for many alkaloids (Matile, 1987; Lichtenthaler, 1999; Morgan and Shanks, 2000; Estevez et al., 2001; Mahmud and Croteau, 2001; Silvestrini et al., 2002), vacuolar membranes might play a pivotal role in compartmentalized acquisition, sequestration and retention of certain secondary metabolites, including alkaloids. Earlier studies have revealed remarkable changes in secondary phytochemical accumulation in the Duboisia myoporoides leaf accompanying its various developmental phases (Mishra and Sangwan, 1996). Besides, to explain the phasic pattern, it has been proposed that 
production and accumulation of secondary phytochemicals require a defined cellular organization and follow a preset developmental programme. This may be correlated with the building-up and maintenance of a cellular endoplasmic system, homeostasis, etc., besides other metabolic factors. Thus, as a part of promising investigations along these lines and their biotechnological applications, it is meaningful to examine the temporal profile of various lipid classes and components with the aim of providing new insights in indole alkaloid accumulation during defined physiological phases of the Rouwolfia serpentina leaf.

\section{Material and Methods}

2.1. Plant material:- Leaf samples were harvested from plants of Rauwolfia serpentina growing in the experimental farm of CIMAP, at Lucknow $\left(26.5^{\circ} \mathrm{N}, 80.5^{\circ} \mathrm{E}, 120 \mathrm{~m}\right.$ a.s.l., subtropical zone), India. Individual leaf buds were tagged on the day of appearance and sampling began $7 \mathrm{~d}$ later. Additional samples were taken at $7 \mathrm{~d}$ intervals until leaf abscission occurred about $77 \mathrm{~d}$ after tagging. The experiments were performed from July to October, a time period characterized by a day length of $12 \pm 1.5 \mathrm{~h}$ and average day and night temperature of approximately 30 and $20^{\circ} \mathrm{C}$, respectively.

2.2. Lipid extraction and analysis: Total lipids were extracted with $\mathrm{CHCl}_{3}-\mathrm{MeOH} 2: 1$ (v/v) and purified with the use of 0.2 volumes of aqueous $0.9 \%(\mathrm{w} / \mathrm{v}) \mathrm{NaCl}$ (Matile, et al., 1987). The purified lipid fraction was evaporated to dryness and the residue weighed. Total fatty acids were determined as previously described (Mishra and Sanwal, 1994).

Lipids were fractionated into neutral, glyco- and phospholipids by S-gel CC eluting in sequence with $\mathrm{CHCl}_{3}$, acetone, and $\mathrm{MeOH}$ (Rouser et al., 1976). Purity of each fraction was checked by bidimensional thin-layer chromatography (TLC) using as solvent systems $\mathrm{CHCl}_{3}-\mathrm{MeOH}-28 \%$ $\mathrm{NH}_{4} \mathrm{OH} \quad$ 65:35:5 (v/v) followed by $\mathrm{CHCl}_{3}$-acetone-MeOH-AcOH-H $2 \mathrm{O} \quad$ 5:2:1:1:0.5 (v/v). The chloroform eluate contained neutral lipids, namely pigments, sterols and nonconjugated fatty acids. The acetone eluate contained glycolipids, whereas the methanol eluate represented mostly phospholipids with traces of glycolipids. Quantitation of each lipid class was performed on the basis of their dry weight determination. Free sterols and nonconjugated fatty acids in the neutral lipid fraction were estimated according to Stadtman (1957) and Lowry and Tinsley (1976), respectively. The glycolipid fraction was freed of trace impurities through TLC on SI-gel G plates employing the solvent system $\mathrm{CHCl}_{3}$-acetone-MeOH-AcOH-H${ }_{2} \mathrm{O}$ 5:2:1:1:0.5 (v/v). Identification of individual glycolipids was carried out by running authentic reference standards simultaneously and also by staining with $\alpha$-naphthol reagent (Siakotes and Rouser, 1965) and water spray (used to facilitate observations of translucent areas on the TLC plates) (Gardner,et al., 1968) was employed for identification and analytical purposes. The separated glycolipids were estimated on the basis of sugar released after hydrolysis (Roughan and Batt, 1968).

2.3. Fatty acid composition: For determination of fatty acid composition, methyl esters were prepared (Jham et al., 1982). Fatty acid methyl esters were separated and detected using an AIMIL-Nucan gas chromatograph fitted with a stainless steel column $(1.8 \mathrm{~m} \mathrm{x} 2 \mathrm{~mm}$, i.d.) of $20 \%$ di-ethylene glycol succinate on Chromosorb $\mathrm{W}(100-120 \mathrm{mesh})$. The operating conditions were as follows: oven temperature, $190^{\circ} \mathrm{C}$; flame ionization detector and injector temperature, $220^{\circ} \mathrm{C}$; flow rate of $\mathrm{H}$ and $\mathrm{N}, 30 \mathrm{~mL}$ min-1. The identification of fatty acid methyl esters was performed by employing authentic reference standards. The peak area was calculated by measuring the height multiplied by the width of the peak at half peak height. The values for each fatty acid are given as percent by weight of total fatty acids.

\section{Results and Discussion}

Specific changes in leaf development beginning from $7 \mathrm{~d}$ after leaf primordial initiation up to abscission include leaf expansion until $28 \mathrm{~d}$, maturation and aging from 35 to $56 \mathrm{~d}$, and senescence initiation by $63 \mathrm{~d}$. Fresh weight and dry weight of leaves increased rapidly during leaf development, attaining maximum values by $35 \mathrm{~d}$ after initiation of bud emergence. Subsequently, leaf weights declined until the end of growth at $77 \mathrm{~d}$. Moisture content of leaf tissue fluctuated from 65 to $90 \%$ (Table 1). One of the characteristic alterations that occur within cells of senescing leaves is the deterioration of membranes. Alterations in cell membrane composition and integrity reflect changes in membrane permeability. Therefore, an understanding of changes in lipid metabolism and lipid content of senescing tissue is fundamental to an understanding of senescence. These complex regulatory cellular and metabolic interactions may influence production, transport and accumulation of indole alkaloids in the developing Rauwolfia serpentina leaf. In the present investigation, changes in lipid content and composition of the Rauwolfia leaf from initiation of leaf emergence to leaf drop have been examined. The changes in moisture percentage appear 
far less prominent than those in fresh and dry weights (Table 1). The profiles up to the $35-42 \mathrm{~d}$ period represent consistent cellular build-up leading to net biomass accumulation. The subsequent period is almost deteriorative in gross metabolic terms. This is substantiated by the observed parallel enhancement in leaf expansion and chlorophyll content (Mishra and Sangwan, 1996). The 35-56 d phase is a characteristic period of leaf maturation and aging phenomenon. The senescence phase (day 56 onwards) accompanies a rapid decrease in chlorophyll level. In fact, breakdown of chlorophyll and a concomitant enhancement in carotenoids are well-established components of senescence (Thomas and Stoddart, 1980).

Table 1: Leaf changes versus development of $R$. serpentina

\begin{tabular}{|l|l|l|l|}
\hline $\begin{array}{l}\text { Leaf Age } \\
(\text { Days })\end{array}$ & $\begin{array}{l}\text { Dresh Weight } \\
(\mathbf{m g} / \text { leaf })\end{array}$ & $\begin{array}{l}\text { Leaf Moisture Content } \\
(\mathbf{m})\end{array}$ \\
\hline 7 & 11.2 & 3.9 & 89 \\
\hline 14 & 23.4 & 10.6 & 83 \\
\hline 21 & 78.5 & 34.6 & 79 \\
\hline 28 & 228.5 & 74.7 & 72 \\
\hline 35 & 582.6 & 165.5 & 65 \\
\hline 42 & 432.7 & 115.6 & 72 \\
\hline 49 & 321.6 & 78.8 & 77 \\
\hline 56 & 295.7 & 67.5 & 79 \\
\hline 63 & 259.9 & 55.6 & 82 \\
\hline 70 & 223.7 & 43.5 & 85 \\
\hline 77 & 182.5 & 35.8 & 90 \\
\hline
\end{tabular}

The total lipid content, expressed as $\mu \mathrm{g}$ per leaf (Table 2), increased during expansion to attain a maximal level of $376 \mu \mathrm{g}$ per leaf at full maturity. However, no marked changes occurred during aging whereas progress of senescence was accompanied by a substantial decline in lipid content. The amount of individual lipid classes per leaf varied grossly in a similar fashion as total lipids except that during aging and senescence initiation the glyco- and phospholipids declined to a far greater extent than the neutral lipids. On the 7th day after proliferation, amongst the lipid classes, neutral lipids were observed to be the most abundant. No consistent pattern of distribution of neutral, glyco- and phospholipids was found up to $28 \mathrm{~d}$ of leaf ontogeny. The average percentages were recorded to be 78,38 and $51.5 \%$, respectively. The period of maturation, aging and senescence accompanied a slow but consistent trend of decline in glyco- and phospholipids. Enhancement in the proportion of neutral lipids during this phase was clearly evident.

Table 2: Total and class wise distributed lipid contents at different developmental stages of $R$. serpentina. Values are mean $\pm S D$ of three sets of experiments with triplicates in each set and expressed as $\mu g / l e a f$.

\begin{tabular}{|l|l|l|l|l|}
\hline $\begin{array}{l}\text { Leaf Age } \\
(\text { Days })\end{array}$ & $\begin{array}{l}\text { Notal Lipids } \\
(\boldsymbol{\mu g} / \mathbf{l e a f})\end{array}$ & $\begin{array}{l}\text { Glycolipids } \\
(\boldsymbol{\mu g} / \mathbf{g} / \mathbf{l e a f})\end{array}$ & $\begin{array}{l}\text { Phospholipids } \\
(\boldsymbol{\mu g} / \mathbf{l e a f})\end{array}$ \\
\hline 7 & $5.2 \pm 0.2$ & $2.9 \pm 0.2$ & $1.7 \pm 0.2$ & $0.9 \pm 0.03$ \\
\hline 14 & $15.9 \pm 0.7$ & $9.8 \pm 0.7$ & $5.4 \pm 0.3$ & $1.1 \pm 0.09$ \\
\hline 21 & $59.8 \pm 1.8$ & $35.8 \pm 1.9$ & $15.7 \pm 0.8$ & $9.5 \pm 0.6$ \\
\hline 28 & $168.5 \pm 3.5$ & $78.2 \pm 2.5$ & $38.3 \pm 1.2$ & $51.5 \pm 0.7$ \\
\hline 35 & $352.8 \pm 5.6$ & $264.2 \pm 3.2$ & $67.3 \pm 2.6$ & $41.8 \pm 1.3$ \\
\hline 42 & $375.8 \pm 3.3$ & $269.4 \pm 3.7$ & $91.1 \pm 2.8$ & $21.2 \pm 0.7$ \\
\hline 49 & $372.6 \pm 3.1$ & $276.4 \pm 3.4$ & $85.1 \pm 2.4$ & $12.9 \pm 1.3$ \\
\hline 63 & $368.4 \pm 2.9$ & $269.5 \pm 3.2$ & $83.2 \pm 1.3$ & $12.4 \pm 0.9$ \\
\hline 70 & $358.5 \pm 2.9$ & $266.4 \pm 3.1$ & $81.3 \pm 1.3$ & $11.3 \pm 0.5$ \\
\hline 77 & $353.2 \pm 2.3$ & $263.1 \pm 2.9$ & $78.2 \pm 1.2$ & $10.9 \pm 0.6$ \\
\hline
\end{tabular}


Overall, the leaf drop stage witnessed a significant increase in neutral lipids and remarkable decline in glycoand phospholipids, as compared to their level in the fully expanded leaf. Specifically, both glycolipids and phospholipids dropped to a greater extent during maturation up to the onset of senescence. It was also noteworthy that the monogalactosyl diglyceride (MGDG)/digalactosyl diglyceride (DGDG) ratio of the Rouwolfia leaf (Table 3) decreased from 5.2 (at complete maturity) to 2.5 (abscised stage), which may be suggestive of substantial changes in ultrastructure, fluidity and permeability of the membrane. An earlier study revealed a greater decrease in MGDG/DGDG ratio in salt-stressed jojoba leaves (Ben-Rais et al., 1993). A report on Duboisia in reference to aging and senescence also entails a significant decrease in MGDG/DGDG ratio (Mishra et al., 1998). It was speculated that the change in their proportion is likely to be correlated with a change in physical properties of organellar membranes (Ben-Rais et al., 1993; Mishra et al., 1998, Mishra et al, 2006). Besides, many studies have revealed that membranes in mature tissues are in liquid crystalline state, while in senescent tissue the membranes or a portion thereof, are in the crystalline gel phase at physiological temperatures (Lees and Thompson, 1980; Chia et al., 1981). Thus, the membrane phase alteration may be a consequence of qualitative changes in membrane lipids like glycolipids, phospholipids and MGDG/DGDG ratio.

Table 3: Alterations in glycolipid composition with respect to developmental differentiation of $R$. serpentina. Values are mean \pm SD of three sets of experiments with triplicates in each set and expressed as $\mu \mathrm{g} / \mathrm{leaf}$. MGDG = Monogalactosyl diglycerides; DGDG $=$ Digalactosyl diglycerides; SQDG = Sulfoquinovosyl diglycerides.

\begin{tabular}{|l|l|l|l|l|}
\hline Leaf Age & MGDG $(\boldsymbol{\mu g} /$ /leaf $)$ & DGDG $(\boldsymbol{\mu g} /$ leaf $)$ & SQDG $(\boldsymbol{\mu g} /$ /eaf $)$ & MGDG/DGDG Ratio \\
\hline 7 & & & & \\
\hline 14 & $0.7 \pm 0.03$ & $0.6 \pm 0.03$ & $0.5 \pm 0.03$ & 1.2 \\
\hline 21 & $3.2 \pm 0.20$ & $1.4 \pm 0.02$ & $0.6 \pm 0.02$ & 2.3 \\
\hline 28 & $11.1 \pm 1.20$ & $3.6 \pm 0.23$ & $0.8 \pm 0.01$ & 3.1 \\
\hline 35 & $28.2 \pm 1.10$ & $7.2 \pm 0.21$ & $2.1 \pm 0.03$ & 3.9 \\
\hline 42 & $50.1 \pm 1.60$ & $11.7 \pm 1.30$ & $4.9 \pm 0.04$ & 4.3 \\
\hline 49 & $66.2 \pm 1.70$ & $14.4 \pm 1.25$ & $9.7 \pm 0.5$ & 4.6 \\
\hline 56 & $63.1 \pm 1.60$ & $12.9 \pm 0.91$ & $8.4 \pm 0.4$ & 4.9 \\
\hline 63 & $61.8 \pm 1.30$ & $11.9 \pm 1.11$ & $8.3 \pm 0.4$ & 5.2 \\
\hline 70 & $61.1 \pm 1.10$ & $13.6 \pm 1.16$ & $7.4 \pm 0.5$ & 4.5 \\
\hline 77 & $57.3 \pm 1.20$ & $14.7 \pm 1.09$ & $6.9 \pm 0.3$ & 3.9 \\
\hline
\end{tabular}

Such spatial alterations in the lipid profile have been reported to enhance membrane permeability (Suttle and Kende, 1978, 1980). The increase in permeability may be, at least partially, due to the occurrence of the crystalline gel phase in the membrane, since both an alteration in permeability and the appearance of the gel phase occur together. These changes in membranes and the resultant alterations in their functions may also be viewed in terms of accumulation/retention of secondary phytochemicals.

These findings may, at least partially, be correlated to changes in alkaloid levels during senescence in $R$. serpentina leaf. Studies are currently underway to determine the spatial mechanism responsible for the sequestration and/or retention of indole alkaloids in leaf tissue. Furthermore, taken together, the current characterization of the lipid profile and studies on gene to metabolic networks for indole alkaloid biosynthesis (Rischer et al., 2006) may be meaningful for biote-chnological research aimed at studying conformational changes in the vacuolar membrane in consequence of growth and senescence and their impact on storage of indole alkaloids. Work along these lines is also in progress.

\section{Acknowledgements}

The present work was supported by a joint venture of the laboratory facility in CIMAP, at Lucknow, India and School of Biotechnology, IFTM University, Moradabad, India. The work embodied in this paper is a part of studies carried out by author (Poonam) registered for her Ph.D. programme at IFTM University, Moradabad, India The authors are grateful to Dr. Surya Prakash Dwivedi (Director, School of Biotechnology, IFTM University, Lodhipur Rajput, Moradabad, U.P., India) for critical reading of the manuscript, helpful advice and valuable discussion. 


\section{References}

[1]. Ben-Rais L, Alpha MJ, Bhal J (1993) Lipid and protein contents of jojoba leaves in relation to salt adaptation. Plant Physiol. 31:547-557.

[2]. Bhatara VS, Sharma JN, Gupta S, Gupta YK. Images in psychiatry. Rauwolfia serpentina: the first herbal antipsychotic. Am J Psychiatry 1997: 154(7): 894.

[3]. Brown JH, Lynch DV, Thompson JE (1987) Molecular species specificity of phospholipids breakdown in microsomal membrane of senescing carnation flowers. Plant Physiol. 85:679-683.

[4]. Canel C, Lopes-Cardoso MI, Whitmer S, van der Fits L, Pasquali G, van der Heijden R, Hoge JH, Verpoorte R (1998) Effects of over-production of strictosidine synthase and tryptophan decarboxylase on alkaloid production by cell cultures of Catharanthus roseus. Planta 205:4-419.

[5]. Chia LS, Thompson JE, Dumbroff EB (1981) Simulation of the effect of leaf senescence on membrane by treatment with paraquat. Plant Physiol. 67:415-420.

[6]. Droillard MJ, Bureau D, Paulin A (1989) Changes in activities of superoxide dismutase during aging of petals of cut carnations (Dianthus Caryophyllus). Physiol. Plant. 76:149-155.

[7]. Duxbury CL, Legge RL, Paliyath G, Barber RF, Thompson JE (1991) Alterations in membrane protein conformation in response to senescence-related changes. Phytochemistry 30:63-68

[8]. Estevez JM, Cantero A, Reindl A, Reichler S, Leon P (2001) 1-deoxy-D-xylulose-5 phosphate synthase, a limiting enzyme for plastidic isoprenoid biosynthesis in plants. J. Biol. Chem. 276:22901-22909.

[9]. Gardner HW (1968) Preparative isolation of monogalactosyl and digalactosyl diglycerides by thin-layer chromatography. Lipid Res. 9:139-141.

[10]. Jham GN, Teles FFF, Campus LG (1982) Use of aqueous hydrochloric acid/MeOH as esterification reagent for analysis of fatty acids. J. Am. Oil Chem. Soc. 59:132-133.

[11]. Klushnichenko VE, Yakimov SY, Tuzova TP, Syagailo YV, Kuzovkina IN, Vulf'son AN, Miroshnikov AI. Determination of indole alkaloids from R. serpentina and R. vomitoria by HPLC and TLC methods. J Chromat 1995; 704: $357-362$.

[12]. Kokate CK, Purohit AP, Gokhale SB. Pharmacognosy. Pune: Nirali Prakashan; 1998; pp 369-373.

[13]. Lowry RR, Tinsley IJ (1976) Rapid colorometric determination of free fatty acids. J. Am. Oil Chem. Soc. 53:470-472

[14]. Lichtenthaler HK (1999) The 1-deoxy-D-xylulose-phosphate pathways of isoprenoid biosynthesis in plants. Annu. Rev. Plant Physiol. Plant Mol. Biol. 50:47-65.

[15]. Mahmoud SS, Croteau RB (2001) Metabolic engineering of essential oil yield and composition in mint by altering expression of deoxyxylulose phosphate reductoiso-merase and menthofuran synthase. Proc. Natl. Acad. Sci. USA. 98:8915- 8920.

[16]. Matile PH, (1987) The sap of plant cells: a review. New Phytol. 105:1-26.

[17]. Mishra S, Sangwan RS (1996) Dynamics of tropane alkaloids in Duboisia myoporoides leaf during development. J. Herbs Spices Med. Plants 4:61-70.

[18]. Mishra S, Shanker S, Sangwan RS (1998) Lipid profile in relation to tropane alkaloid production and accumulation during leaf growth and senescence in Duboisia myoporoides. Fitoterapia 69:65-72.

[19]. Mishra S, Sanwal GG (1994) Effect of Cuscuta infection on chloroplast lipid composition of Brassica leaves. Eur J. Plant Pathol. 100:61-70.

[20]. Mishra, S., Tyagi, A., Singh, I.V. and Sangwan, R.S. (2006). Studies on alterations in lipid profile during growth and senescence of Catharanthus roseus leaf. Brazilian Journal of Plant Physiology 18 (4): 447-454.

[21]. Morgan JA, Shanks JV (2000) Determination of metabolic rate limitations by precursor feeding in Catharanthus roseus hairy root culture. J Biotechnol. 79:137-145.

[22]. Pullaiah J. Medicinal plants in India, Vol. II. New Delhi: Regency Publ; 2002; pp 441-443.

[23]. Rischer H, Oresic M, Seppänen-Laakso T, Katajamaa M, Lammertyn F, Ardiles-Diaz W, Van Montagu MCE, Inzé D, Oksman-Caldentey K-M, Goossens A (2006) Gene to metabolite networks for terpenoid indole alkaloid biosynthesis in Catharanthus roseus cells. Proc. Natl. Acad. Sci. USA 103:5614-5619.

[24]. Roughan PG, Batt RD (1968) Quantitative analysis of sulpholipids, sulphoquinovosyl diglycerides and galactolipids: Monogalactosyl diglycerides and digalactosyl diglycerides in plant tissues. Anal. Biochem. 22:74-78.

[25]. Rouser G, Kritchevsky G, Yamamoto A (1976) Column chromatographic and associated procedures for separation and determination of phospholipids and glycolipids. In: Marinetti GV (ed), Lipid Chromatographic Analysis, Vol. 3, pp.99-162, Marcel Dekker, New York.

[26]. Siakotes AN, Rouser G (1965) Analytical separation of non-lipid soluble substances and gangliosides from other lipids by dextran gel column chromatography. J. Am. Oil. Chem. Soc. 42:913-919.

[27]. Silvestrini A, Pasqua G, Botta B, Monacelli B, van der Heijden R, Verpoorte R (2002) Effect of alkaloid precursor feeding on aCamptotheca acuminate cell line. Plant Physiol. Biochem. 40:749-753.

[28]. Stadtman TC (1957) Preparation and assay of cholesterol and ergosterol. Meth. Enzymol. 3:392-394.

[29]. Stanford JL, Martin EJ, Brinton LA, Hoover RN. Rauwolfia use and breast cancer: A casecontrol study. J Natl Cancer Inst 1986; 76: 817-822.

[30]. Suttle JC, Kende H (1978) Ethylene and senescence in petals of Tradescantia. Plant Physiol. 62:267-271. Thomas H, Stoddart JL (1980) Leaf senescence: a review. Annu. Rev. Plant Physiol. 31:83-111.

[31]. Lees G.L., Thompson J.E. (1980) Lipid composition in plasma membrane enriched fractions from senescing cotyledons. Physiol. Plant. 49:215-221.

[32]. Von Poser G, Andrade HH, Da Silva KV, Henriques AT, Henriques JA. Genotoxic, mutagenic and recombinogenic effects of Rauwolfia alkaloids. Mutat Res 1990; 232: 37-43. 